Article

\title{
Assessment of the KID Procedure on a Mo-oxo Complex, an Open-Shell System
}

\author{
Jorge I. Martínez-Araya ${ }^{1, \ddagger * * \mathbb{D}}$, Daniel Glossman-Mitnik $2, \ddagger(\mathbb{D}$ \\ 1 Departamento de Ciencias Químicas, Facultad de Ciencias Exactas, Universidad Andres Bello (UNAB), Av. \\ República 498, Santiago-Chile; jorge.martinez@unab.cl \\ 2 Laboratorio Virtual NANOCOSMOS, Departamento de Medio Ambiente y Energía, Centro de Investigación \\ en Materiales Avanzados, Chihuahua, Chih 31123, Mexico; daniel.glossman@cimav.edu.mx \\ * Correspondence: jorge.martinez@unab.cl; Tel.: +56-2277-039-57 (F.L.) \\ $\ddagger$ These authors contributed equally to this work. \\ Version July 31, 2020 submitted to Journal Not Specified
}

\begin{abstract}
The KID (Koopmans in DFT) procedure usually applies in organic molecules of the closed-shell type. We used the KID procedure in an open-shell system for the first time to choose the most suitable density functional to compute global and local reactivity descriptors coming from the Conceptual Density-Functional Theory. From a set of 18 density functionals spread from the second until the fourth rung of the Jacob's ladder: BP86, B97-D, BLYP, CAM-B3LYP, M06-L, M11-L, MN12-L, B3LYP, PBE0, N12-SX, M06-2X, M11, MN12-SX, CAM-B3LYP, LC- $\omega$ HPBE, $\omega$ B97X-D, APFD, MN15 and MN15-L, we concluded that CAM-B3LYP provides the best outcome.
\end{abstract}

Keywords: KID protocol; Global Reactivity; Conceptual DFT; Kopmans' Theorem; Mo-oxo Complexes; Hydrogen production

\section{Introduction}

Molybdenum, a second-row transition metal, has become a promising candidate to synthesize new catalysts. Specifically, the scientific literature revealed findings involving this metal in $\mathrm{H}_{2}$ production from water scission[1,2]. Karunadasa et al.[3] found a Mo-N-based metal-organic compound whose molecular formula is given by $\left[\left(\mathrm{PY}_{5} \mathrm{Me}_{2}\right) \mathrm{Mo}\left(\mathrm{CF}_{3} \mathrm{SO}_{3}\right)\right]^{+}$, being $\mathrm{PY}_{5} \mathrm{Me}_{2}$ the pentadentate ligand 2,6-bis[1,1-bis(2-pyridil)ethyl]-pyridine.[4].

The global process is summarized by the following chemical reaction:

$$
\left[\left(\mathrm{PY}_{5} \mathrm{Me}_{2}\right) \mathrm{Mo}\left(\mathrm{CF}_{3} \mathrm{SO}_{3}\right)\right]^{+} \underset{-\mathrm{CF}_{3} \mathrm{SO}_{3}}{\stackrel{+\mathrm{H}_{2} \mathrm{O}}{\longrightarrow}}\left[\left(\mathrm{PY}_{5} \mathrm{Me}_{2}\right) \mathrm{MoO}\right]^{2+}+\mathrm{H}_{2}
$$

These researchers experimentally confirmed that $\left[\left(\mathrm{PY}_{5} \mathrm{Me}_{2}\right) \mathrm{Mo}\left(\mathrm{H}_{2} \mathrm{O}\right)\right]^{2+}$ is able to turn water into $\mathrm{H}_{2}$ through a suggested catalytic cycle[3] where the $\left[\left(\mathrm{PY}_{5} \mathrm{Me}_{2}\right) \mathrm{Mo}(\mathrm{H})(\mathrm{OH})\right]^{+}$complex can release $\mathrm{H}_{2}$, as a result the $\left[\left(\mathrm{PY}_{5} \mathrm{Me}_{2}\right) \mathrm{MoO}\right]^{+}$complex has the feature of capturing electrons through multiple steps, leading to the aquo-Mo complex which makes it an electrocatalyst to release $\mathrm{H}_{2}$ in neutral aqueous media and seawater. Therefore the $\left[\left(\mathrm{PY}_{5} \mathrm{Me}_{2}\right) \mathrm{MoO}\right]^{+}$complex is the focus of our attention as Figure 1 depicts. 


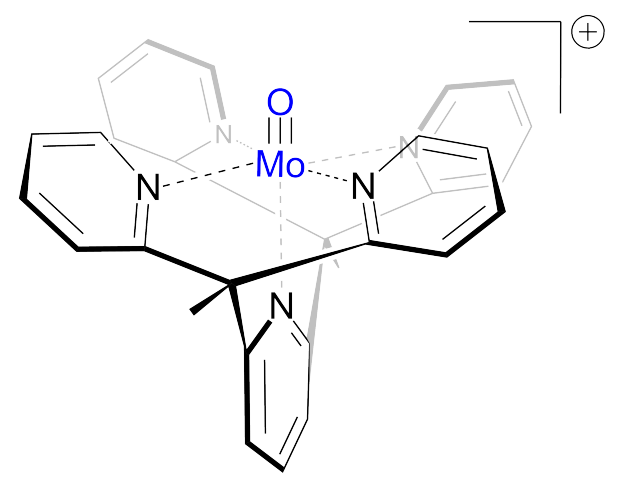

Figure 1. $\left[\left(\mathrm{PY}_{5} \mathrm{Me}_{2}\right) \mathrm{MoO}\right]^{+}$complex.

The most recent investigation concerning compounds based on $\left[\left(\mathrm{PY}_{5} \mathrm{Me}_{2}\right) \mathrm{MoO}\right]^{+}$complexes was aimed to analyze the electron-withdrawing and electron-donating nature of substituent groups along with their locations at para-position of equatorial/axial pyridine rings in order to study its influence on kinetics $\left(\Delta E^{\ddagger}\right)$ and thermodynamics $\left(\Delta E^{\circ}\right)$ of the molecular hydrogen release process. The results revealed an opposite effect given by each type of substituent group depending on if located on equatorial or axial pyridine rings[5]. Furthermore, a difference between electron-withdrawing or electron-donating substituent groups at axial para-positions can be distinguished visually utilizing the so-called dual descriptor better than spin-density[6].

An analysis of the global and local reactivity of Mo-oxo complexes as electrocatalysts is mandatory to attain a deep understanding of the molecular hydrogen release process[7,8]. Previous theoretical studies focused on: the energy barrier, overall energy, substituent effect, explicit and implicit solvent effect, along with the use of few local reactivity descriptors. All of them allowed to gain more insights concerning this type of electrocatalyst[3,5,7]. However, all these theoretical approaches have assumed none dependence of the density functional, i.e. up to now we assumed the BP86 density functional is accurate enough to carry out these analyses. Karunadasa et al.[3] suggested the use of BP86 owing its best performance to reproduce energetic values, which are the closest to those obtained experimentally in agreement with electrochemical measurements. Nevertheless, reactivity descriptors usually do not link in the same way, so being necessary to assess a representative set of density functionals to unveil whether BP86 is still proper to evaluate reactivity or not in the framework of the Conceptual-DFT (CDFT)[9,10]. Besides, this study is required because condensed values of local reactivity descriptors implemented in some quantum chemical or post-SCF softwares are based on the frontier molecular orbital approximation, meaning that HOMO and LUMO drive the complete reactivity of a molecule. On the other hand, electronic chemical potential and molecular hardness, the main two global reactivity descriptors coming from the CDFT, are usually computed according to the Koopmans' theorem.

In the present work, we used the KID procedure proposed by Glossman-Mitnik et al.[11-14] where a set of indexes called J quantify deviations when using the Koopmans' theorem. In order to quantify a suitable density functional to be used in computing global reactivity descriptors and since the number of available density functionals is too large as to perform an analysis functional by functional[15-17], we analyzed 18 density functionals spread in the first four rungs of Jacob's ladder. It is worthwhile to mention this is an alternative procedure to validate the application of the Koopmans' theorem different that one proposed by Bellafont et al.[18] where the biding energies assess the validity of the Koopmans' theorem. 


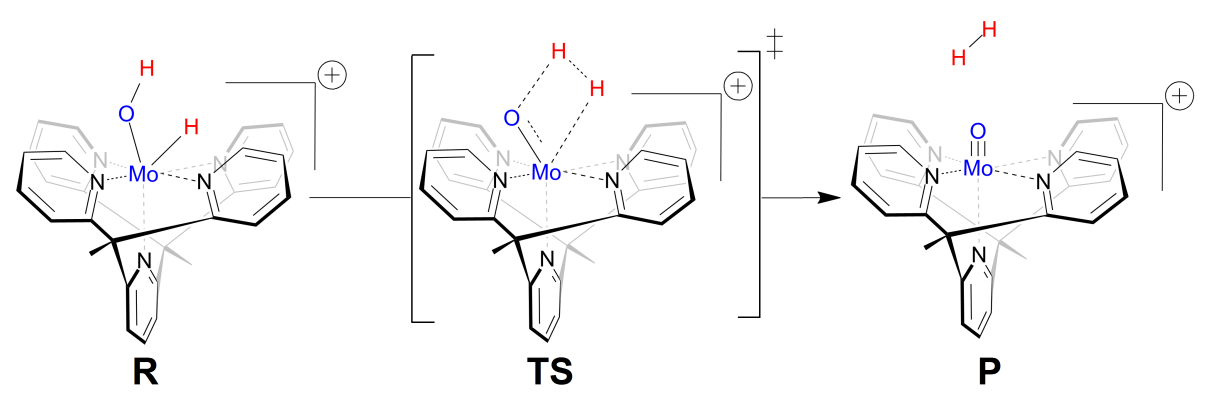

Figure 2. Reactant (R), transition state (TS) and product (P) in the key step that releases molecular hydrogen. The $\left[\left(\mathrm{PY}_{5} \mathrm{Me}_{2}\right) \mathrm{MoO}\right]^{+}$complex is produced and corresponds to the system under analysis in the present work.

\section{Theoretical Background}

The review of the results obtained in the study was aimed at confirming the fulfillment of the KID (Koopmans in DFT) protocol on an open-shell system. On doing it previously, several descriptors associated with the results that the HOMO and LUMO calculations obtained are related with results coming from the vertical first ionization potential $(I)$ and vertical first electron affinity (A) following the $\triangle \mathrm{SCF}$ procedure, where SCF refers to the Self-Consistent Field technique. There is a connection between the three key descriptors and the simplest conformity to the theorem of Koopmans by connecting $\epsilon_{H}$ to $-I, \epsilon_{L}$ to $-A$, and their actions by defining the HOMO-LUMO gap $J_{I}=\left|\epsilon_{H}+E_{g s}(N-1)-E_{g s}(N)\right|, J_{A}=\left|\epsilon_{L}+E_{g s}(N)-E_{g s}(N+1)\right|$, and $J_{H L}=\sqrt{J_{I}^{2}+J_{A}^{2}}$. It should be noticed that the $J_{A}$ descriptor consists of an approximation which is only valid if the HOMO of the radical anion (the SOMO in the system with $N+1$ electrons) resembles the LUMO of the neutral system, then the use of $J_{A}$ makes sense while LUMO tending to SOMO. For this reason, another descriptor $|\Delta S L|$ has been designed [11-14], to help in the verification of the accuracy of the approximation. Notice that a $\Delta S L \longrightarrow 0$ does not guarantee the fulfillment of the Koopmans's theorem, $|\Delta S L|$ is a control parameter for the $J_{A}$ so discarding a possible $J_{A}=0$ which could be just a coincidence when $|\Delta S L| \neq 0$. In molecules like mirabamides [19] $|\Delta S L|<0.03$; however, we cannot expect similar magnitudes when dealing with metal-organic or organometallic molecules as the Mo-oxo complex analyzed in the present work; in consequence, this article marks a precedent in the use of the KID protocol on open-shell metal-transition complexes.

It should be noticed that if the HOMO of the radical anion (the SOMO in the system with $N+1$ electrons) resembles the LUMO of the neutral system, then $J_{A}$ descriptor is a valid approximation, hence the use of $J_{A}$ makes sense while LUMO tending to SOMO. To sum up, the lower the $J$ values, the more suitable the Koopman's theorem application is. Since $J=0$ is a too hard condition to meet, then we propose a $J$ threshold value equals 0.5 to select the most proper density-functional; while $|\triangle S L|$ is expected to be so small as possible.

The Koopmans' theorem[20] should be applied only within the context of the Hartree-Fock's theory and not DFT. Nevertheless, Toro-Labbé and Zevallos demonstrated that electronic chemical potential, $\mu=-0.5(I+A)$, and molecular hardness, $\eta=I-A$, based on Kohn-Sham's frontier molecular orbitals yield the same trends given by the same global reactivity descriptors based on frontier molecular orbitals of the Hartree-Fock theory[21]. The exact physical meaning could assign to the Kohn-Sham HOMO using the "Kohn-Sham analog of Koopmans' theorem in the Hartree-Fock theory", meaning that the Kohn-Sham HOMO approximately equals the opposite of the first vertical ionization potential, $-I[22]$. The Janak's theorem[23] backs up this statement. 


\section{Results}

All results are quoted in Table 1.

Table 1. HOMO, LUMO and SOMO orbital energies, HOMO-LUMO gap and the KID descriptors (all in $\mathrm{eV}$ ) tested in the verification of the Koopmans-like behavior of the CAM-B3LYP density functional for the Mo complex. The (1) and (2) represent the $6-31+G(d, p)$ and $6-311+G(d, p)$ basis sets, respectively.

\begin{tabular}{|c|c|c|c|c|c|c|c|c|}
\hline DF & HOMO & LUMO & SOMO & H-L Gap & $J(I)$ & $J(A)$ & $J(H L)$ & $|\Delta \mathrm{SL}|$ \\
\hline \multicolumn{9}{|l|}{ GGA } \\
\hline BP86(1) & -5.9076 & -5.6475 & -2.5396 & 0.2601 & 1.682 & 1.574 & 2.304 & 3.108 \\
\hline BP86(2) & -5.9764 & -5.7160 & -2.6020 & 0.2604 & 1.683 & 1.581 & 2.309 & 3.114 \\
\hline B97-D(1) & -5.7128 & -5.4589 & -2.3418 & 0.2539 & 1.681 & 1.594 & 2.316 & 3.117 \\
\hline B97-D(2) & -5.7773 & -5.5187 & -2.3968 & 0.2544 & 1.681 & 1.600 & 2.321 & 3.122 \\
\hline BLYP(1) & -5.6227 & -5.3680 & -2.2825 & 0.2547 & 1.679 & 1.559 & 2.291 & 3.086 \\
\hline BLYP(2) & -5.6883 & -5.4338 & -2.3402 & 0.2544 & 1.681 & 1.567 & 2.298 & 3.094 \\
\hline \multicolumn{9}{|l|}{ MGGA } \\
\hline M06-L(1) & -5.6491 & -5.2896 & -2.2444 & 0.3595 & 1.637 & 1.541 & 2.249 & 3.045 \\
\hline M06-L(2) & -5.7223 & -5.3604 & -2.3081 & 0.3619 & 1.639 & 1.5550 & 2.256 & 3.052 \\
\hline M11-L(1) & -6.0358 & -5.7389 & -2.6145 & 0.2969 & 1.673 & 1.622 & 2.330 & 3.124 \\
\hline M11-L(2) & -6.0526 & -5.7661 & -2.66664 & 0.2865 & 1.681 & 1.608 & 2.327 & 3.100 \\
\hline MN12-L(1) & -5.6472 & -5.1800 & -2.2300 & 0.4672 & 1.596 & 1.489 & 2.182 & 2.950 \\
\hline MN12-L(2) & -5.7114 & -5.2379 & -2.2844 & 0.4735 & 1.593 & 1.499 & 2.187 & 2.954 \\
\hline MN15-L(1) & -5.7296 & -5.3419 & -2.2977 & 0.3878 & 1.635 & 1.587 & 2.279 & 3.044 \\
\hline MN15-L(2) & -5.7767 & -5.3876 & -2.3883 & 0.3891 & 1.636 & 1.570 & 2.268 & 2.999 \\
\hline \multicolumn{9}{|l|}{ HGGA } \\
\hline B3LYP(1) & -6.0349 & -4.6744 & -2.5356 & 1.3606 & 1.181 & 1.072 & 1.595 & 2.139 \\
\hline B3LYP(2) & -6.1035 & -4.7302 & -2.5932 & 1.3734 & 1.181 & 1.070 & 1.594 & 2.137 \\
\hline PBE0(1) & -6.1593 & -4.5125 & -2.5856 & 1.6468 & 1.045 & 0.980 & 1.433 & 1.927 \\
\hline PBE0(2) & -6.2271 & -4.5623 & -2.6420 & 1.6648 & 1.043 & 0.976 & 1.428 & 1.927 \\
\hline N12-SX(1) & -5.7255 & -4.8172 & -2.2134 & 0.9083 & 1.398 & 1.308 & 1.914 & 2.604 \\
\hline N12-SX(2) & -5.8034 & -4.8733 & -2.2735 & 0.9301 & 1.397 & 1.305 & 1.912 & 2.599 \\
\hline \multicolumn{9}{|l|}{ HMGGA } \\
\hline M06-2X(1) & -6.5944 & -3.5590 & -3.3315 & 3.0354 & 0.309 & 0.320 & 0.445 & 0.228 \\
\hline M06-2X(2) & -6.7261 & -3.5927 & -3.4975 & 3.1334 & 0.266 & 0.291 & 0.395 & 0.095 \\
\hline M11(1) & -8.1338 & -2.6079 & -3.4509 & 5.5258 & 1.245 & 0.657 & 1.247 & 0.843 \\
\hline M11(2) & -7.4763 & -2.5905 & -4.0619 & 4.8858 & 0.576 & 0.619 & 0.845 & 1.471 \\
\hline MN12-SX(1) & -5.8638 & -4.9659 & -2.4105 & 0.8979 & 1.395 & 1.272 & 1.888 & 2.555 \\
\hline MN12-SX(2) & -5.9438 & -5.0224 & -2.4727 & 0.9214 & 1.391 & 1.274 & 1.887 & 2.550 \\
\hline MN15(1) & -6.3324 & -4.4099 & -2.8713 & 1.9225 & 0.498 & 0.675 & 0.839 & 1.539 \\
\hline MN15(2) & -6.6140 & -4.0357 & -2.9636 & 2.5783 & 0.621 & 0.554 & 0.832 & 1.072 \\
\hline \multicolumn{9}{|l|}{ LC-DFT } \\
\hline CAM-B3LYP(1) & -7.0524 & -3.5710 & -3.8414 & 3.4814 & 0.168 & 0.276 & 0.323 & 0.271 \\
\hline CAM-B3LYP(2) & -6.8780 & -3.4014 & -3.1005 & 3.4765 & 0.148 & 0.198 & 0.247 & 0.301 \\
\hline LC- $\omega \mathrm{HPBE}(1)$ & -7.6899 & -3.4948 & -4.3163 & 4.1952 & 0.975 & 1.786 & 2.034 & 0.822 \\
\hline LC- $\omega \mathrm{HPBE}(2)$ & -7.8494 & -2.4667 & -4.4371 & 5.3827 & 0.787 & 0.676 & 1.038 & 1.970 \\
\hline$\omega \mathrm{B} 97 \mathrm{X}-\mathrm{D}(1)$ & -7.2608 & -2.7369 & -3.5043 & 4.5239 & 0.390 & 0.339 & 0.517 & 0.767 \\
\hline$\omega \mathrm{B} 97 \mathrm{X}-\mathrm{D}(2)$ & -7.3297 & -2.7815 & -3.5620 & 4.5481 & 0.397 & 0.349 & 0.528 & 0.780 \\
\hline APFD(1) & -6.1740 & -4.6172 & -2.6188 & 1.5568 & 1.101 & 0.997 & 1.485 & 1.998 \\
\hline $\operatorname{APFD}(2)$ & -6.2418 & -4.6700 & -2.6768 & 1.5717 & 1.099 & 0.993 & 1.481 & 1.993 \\
\hline
\end{tabular}




\section{Discussion}

First of all, we must focus our attention on the $|\Delta S L|$ value because it permits us to discard or accept the application of the KID protocol according to $J(H L)$ values for a certain density functional. Then we can define a threshold value for $|\Delta S L|$, e.g., 1.0 so that all those density functionals resulting in $|\Delta S L|>1.0$ should be discarded because, in such cases, $J_{A}$ values make no sense since each one indicates that LUMO differs from SOMO as established by the threshold aforementioned. Even so, we included in discussion all obtained values since this is the first time that this protocol being applied on a transition-metal complex of the open-shell type.

The GGA density functionals (DF) present the highest deviations from the Koopmans' theorem with $2.298<J(H L)<2.321$ along with the average values of $\langle J(H L)\rangle_{(1)}=2.304$ and $\langle J(H L)\rangle_{(2)}=2.309$ where (1) and (2) represent the $6-31+\mathrm{G}(\mathrm{d}, \mathrm{p})$ and $6-311+\mathrm{G}(\mathrm{d}, \mathrm{p})$ basis sets, respectively. Additionally this performance becomes worse when the size of basis set increases. The BLYP is the best DF in this set with the $6-31+G(d, p)$ basis set for non metal atoms.

The MGGA set of DF also shows similar deviations from the Koopmans' theorem since that $2.182<J(H L)<2.330$ and the average values of $\langle J(H L)\rangle_{(1)}=2.260$ and $\langle J(H L)\rangle_{(2)}=2.260$. Besides we observe that the increase of the basis set size do not improve the performance of the Koopmans' theorem. The 6-311+G(d,p) basis set for non metal atoms only favor the $J(I)$ when using MN12-L, but in general the best DF in the MGGA set is MN12-L along when using the $6-31+G(d, p)$ basis set for non metal atoms. The HGGA set of DF shows us a more significant improvement because $1.428<J(H L)<1.914$ and the average values of $\langle J(H L)\rangle_{(1)}=1.647$ and $\langle J(H L)\rangle_{(2)}=1.645$, being the PBE0 the best DF with the 6-311+G(d,p) basis set for non metal atoms.The HMGGA set of DF presents a better performance in the use of the Koopmans' theorem when comparing with the MGGA set because $0.395<J(H L)<1.888$ and $\langle J(H L)\rangle_{(1)}=1.105$ and $\langle J(H L)\rangle_{(2)}=0.990$, where M06-2X is the best $\mathrm{DF}$ along with the $6-311+\mathrm{G}(\mathrm{d}, \mathrm{p})$ basis set.

The noticeable improvement comes from the LC-DFT set because $0.247<J(H L)<2.034$ and $\langle J(H L)\rangle_{(1)}=0.958$ and $\langle J(H L)\rangle_{(2)}=0.604$, being the CAM-B3LYP not only the best DF in this set, but also in all sets considered in the present study. One explanation could be based on the construction of this functional: On the one hand, CAM-B3LYP is composed of 0.19 Hartree-Fock (HF) plus 0.81 Becke 1988 (B88) exchange interaction at short-range. On other hand, it has $0.65 \mathrm{HF}$ plus 0.35 B88 at long-range and finally the standard error function describes the intermediate region. As we observe, this result is related to the presence of an amount of HF exchange interaction, but not with the inclusion of dispersion terms since CAM-B3LYP has not them in its mathematical formulation. Evidence that favors this claim is detected through the remarkable difference between MN15 and MN15-L, clearly the inclusion of Hartree-Fock exchange improves the performance of $J$ indexes. All those density functionals lack of HF exchange (0\%) lead to worse $J$ values: BP86, B97-D, BLYP, M06-L, M11-L, MN12-L, MN15-L. After including the HF exchange, $J$ values improve as: B3LYP (20\%), $\omega$ B97X-D $(22.2 \%)$, PBE0 (25\%), N12-SX (25\%), MN12-SX (25\%), M11 (42.8\%), MN15 (44\%), and M06-2X (54\%). Notice that we also included results coming from the use of the APFD density functional having a $23 \%$ of HF exchange; it shows us better results than given by DFs lacking HF exchange, but similar that is offered by density-functionals of the HGGA-type. 
However, the improvement in the performance of the Koopmans' theorem by a density functional must be constrained the split of the interelectronic Coulomb operator into a short-range part and a long-range part, having the latter a more percentage of HF exchange. Now the focus of our attention should be paid on the those density functionals including this separation of the interelectronic Coulomb operator and weighting the HF exchange at short- and long-range. In the case of MN11 we have $42.8 \%$ (short-range) and $11 \% \mathrm{X}$ (long-range); CAM-B3LYP, 19\% (short-range) and 65\% (long-range); LC- $\omega \mathrm{HPBE}, 0 \%$ (short-range) and $40 \%$ (long-range); and $\omega \mathrm{B} 97 \mathrm{X}-\mathrm{D}, 22.2 \%$ (short-range) and $100 \%$ (long-range). The LC- $\omega$ HPBE presents the worst performance among the LC-DFT set of density functionals due to its lacking of short-range HF exchange $(J(H L)=2.034$ with the $6-31+\mathrm{G}(\mathrm{d}, \mathrm{p})$ basis set and $J(H L)=1.038$ with the $6-311+\mathrm{G}(\mathrm{d}, \mathrm{p})$ basis set). When one increases the basis set, the performance of LC- $\omega$ HPBE worsens since that despite being $J(H L)=1.038$, the $|\Delta S L|=1.970$.

Additional evidence supporting the use of the CAM-B3LYP density functional that has to do with the HOMO-LUMO gap comes from the benchmark study performed by Tecmer et al.[24] Since Time-Dependent DFT (TD-DFT) results in excited states that sometimes appear lower in energy when compared against the reference state, they applied TD-DFT with the following set of density functionals: LDA, PBE, BLYP, B3LYP, PBE0, M06, M06-L, M06-2X, CAM-B3LYP. Vertical excitation energies obtained were compared with reference data obtained using accurate wave-function theory (WFT) methods for the electronic spectrum of the $\mathrm{UO}_{2}{ }^{2+}$. As a result, they found that CAM-B3LYP was able to produce the best agreement in comparison to coupled-cluster data, thus revealing the importance of proper inclusion of HF exchange as offered by a hybrid functional like CAM-B3LYP in transition-metal compounds. In fact, for the CAM-B3LP functional, $|\Delta S L|=0.301$, implies that SOMO differs from LUMO by about less than $9 \%$, so that the variation from $|\Delta S L|=0.271$ up to $|\Delta S L|=0.301$ when changing the basis set from $6-31+G(\mathrm{~d}, \mathrm{p})$ to $6-311+\mathrm{G}(\mathrm{d}, \mathrm{p})$ is not significant.

Finally, as $|\Delta S L|$ indicates, GGA, MGGA, and HMGGA density functionals cannot be used to apply global reactivity descriptors coming from the Conceptual DFT when the Koopmans' theorem is applied. The reader should have in mind that the use of the Koopmans' theorem allows one to obtain values of global or even local reactivity descriptors because a fast analysis of reactivity through the use of global descriptors of the CDFT is carried out firstly through of the Koopmans' theorem. Then $J$ indexes provide us quantitative criteria to validate whether the Koopmans' theorem is suitable to be used within this context or not.

\section{Settings and Computational Methods}

We used the Gaussian09[25] suite of programs for 16 density functionals excepting MN15 and MN15-L that were employed through the use of the Gaussian16[26] quantum chemistry package. The following exchange-correlation functionals[15-17] were used:

GGA-type: BP86, B97-D (D meaning thus an addition of molecular mechanic dispersion corrections), and BLYP.

MGGA-type: M06-L, M11-L, and MN12-L (non-separable gradient approximation).

HGGA-type: B3LYP, PBE0, and N12-SX (range-separated hybrid).

HMGGA-type: M06-2X, M11, and MN12-SX (range-separated hybrid).

LC-DFT-type: CAM-B3LYP, LC- $\omega$ HPBE, $\omega$ B97X-D (range-separated hybrid), MN15, and MN15-L. 
Exclusively of Gaussian: APFD (Austin-Frisch-Petersson functional with dispersion)[27]

Besides we included the effective core potential MWB28[28-31] for metal center and the standard Gaussian-type orbitals 6-31+G(d,p) and 6-311+G(d,p)[32-35,35-37] basis sets for N, C, O, and $\mathrm{H}$ atoms. For each level of theory, we performed geometrical optimizations on the molecular system with its original number of electrons $(N)$. Afterward, each optimized geometry was used in single-point calculations with $N+1$ and $N-1$ electrons in order to compute the vertical first ionization potential $(I)$ and the vertical first electron affinity $(A)$. Finally, we compared couples $I$ and $A$ against couples HOMO and LUMO energies through the use of the $J$ indexes. We carried out harmonic vibrational frequencies analyses to confirm the obtained structures correspond to minima on the Born-Oppenheimer surface: we did not find imaginary frequencies on the $\mathrm{N}$-electron geometrical optimized structure for each used density functional.

\section{Conclusions}

Nowadays, there are more than 200 density functionals available to execute quantum chemical calculations. Depending upon the goal established by users, certain functionals are more appropriate than others to execute quantum chemical calculations. In the context of Conceptual DFT, the choice of a density functional sometimes is an issue of personal preference based on specific criteria that could have nothing to do with the analysis of molecules reactivity. In this work, we have applied the KID (Koopmans' in DFT) procedure on an open-shell metal-organic cation complex for the first time to decide the best density functional from the Koopmans' theorem point of view to use reactivity descriptors from the Conceptual DFT. From the set of density functionals we selected, the best one corresponds to CAM-B3LYP, thus discarding the use of BP86 to perform any analysis concerning reactivity and selectivity through the use of Conceptual-Density-Functional Theory. An energetic difference as $9 \%$ between SOMO and LUMO is acceptable to validate the applied criterion given by the KID protocol. Since this is not the only transition metal complex to be analyzed, the next calculations will involve more based-Mo complexes participating in the catalytic cycle that supports the mechanism explaining the molecular hydrogen release. Alike, a similar analysis will be performed on the reactant and transition state from the chemical reaction given by Figure 2. Another reason for using the KID protocol is based on the fact that some programs of Quantum Chemistry use the Frontier Molecular Orbital Approximation to compute values of local reactivity descriptors. That means that HOMO and LUMO energies are not only input data to compute global reactivity through global reactivity descriptors as chemical potential and molecular hardness [9,10], but also Fukui functions $[9,10]$ and dual descriptor[38-40] employ the squares of HOMO and LUMO as a first approximation to reveal the most susceptible sites to undergo nucleophilic and electrophilic sites of molecules.

Author Contributions: Conceptualization, D.G. and J.M.; methodology, D.G.; software, D.G. and J.M.; validation, D.G. and J.M.; formal analysis, J.M. and D.G.; investigation, J.M.; resources, D.G. and J.M.; writing-original draft preparation, J.M. and D.G.; writing-review and editing, J.M.; visualization, J.M.; supervision, J.M.; project administration, J.M.; funding acquisition, J.M. All authors have read and agreed to the published version of the manuscript.

Funding: This research was funded by FONDECYT grant number 1181504, whose support is acknowledged by Jorge I. Martínez-Araya.

Acknowledgments: Daniel Glossman-Mitnik is a Researcher from CONACYT and CIMAV from which partial support is gratefully acknowledged

Conflicts of Interest: The authors declare no conflict of interest. The funders had no role in the design of the study; in the collection, analyses, or interpretation of data; in the writing of the manuscript, or in the decision to publish the results. 


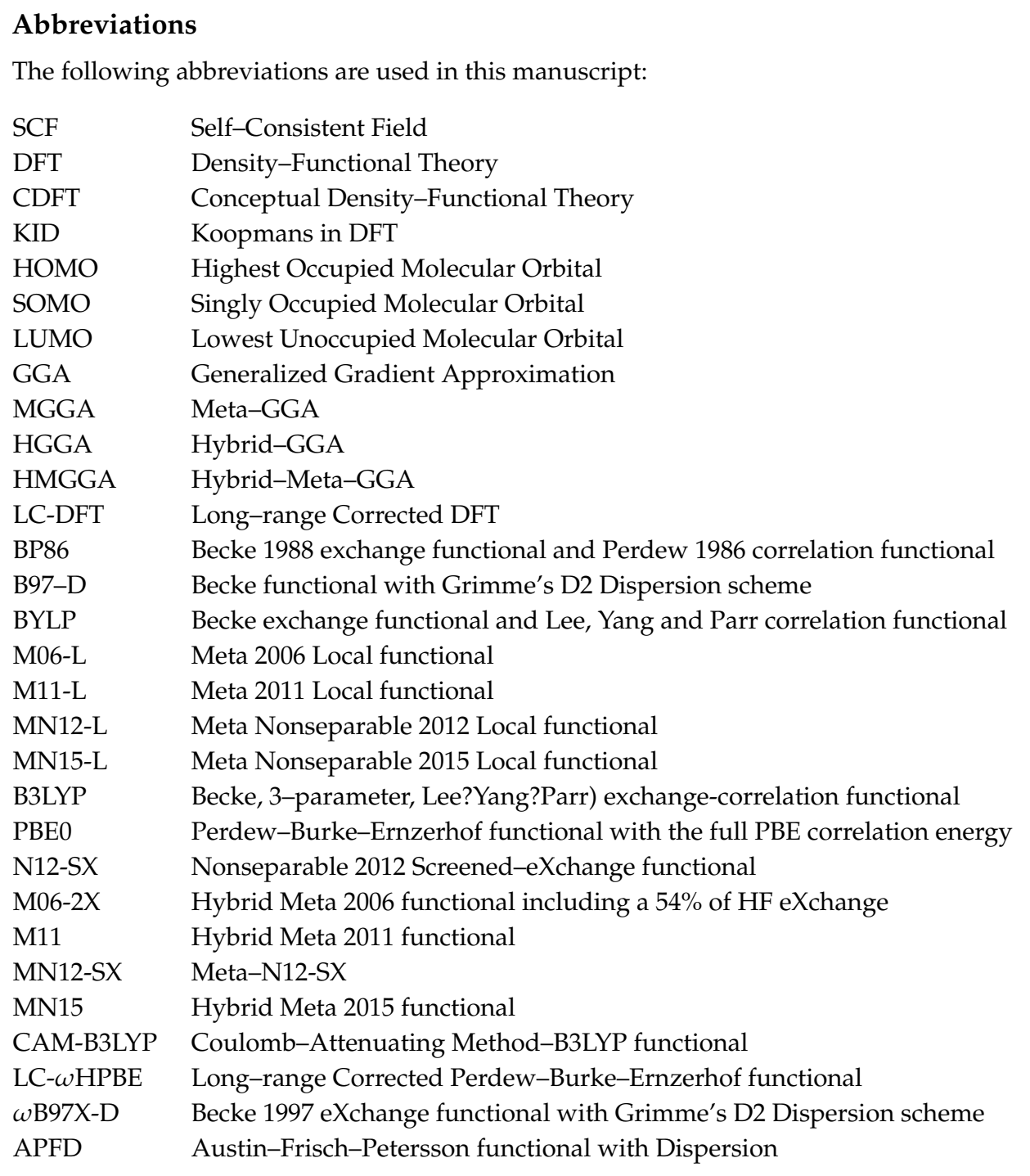

\section{Appendix A How to obtain the LC- $\omega$ HPBE density functional in Gaussian 09.}

Since the LC- $\omega$ HPBE is absent in Gaussian 09, the use of internal Options provided by this suite of programs allows one to use that density functional as follows:

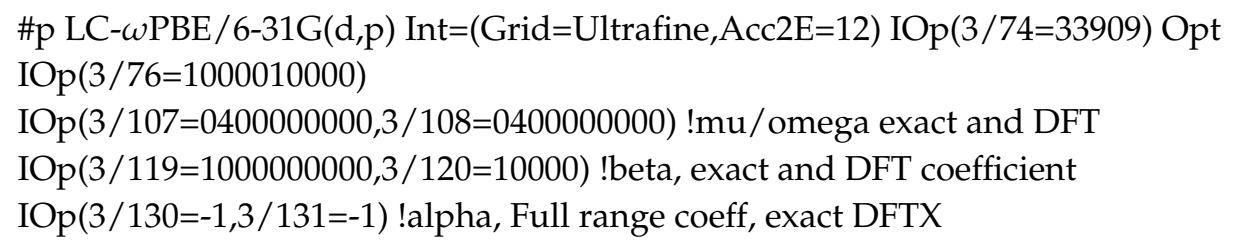

It will provide the equivalent of the LC- $\omega \mathrm{HPBE}$ density functional.

$\operatorname{IOp}(3 / 74=33909)$ replace the functional, but having LC $-\omega \mathrm{PBE}$ sets up the required structure for the LC treatment of a hybrid functional. The user has to bear in mind that the IOp structure is not passed between multi-step jobs. So if a frequency calculation is required, a second job step must be performed repeating the IOps and without using the Opt Freq, but only Freq. Notice that the integration thresholds which are used with G16 must be specified in Gaussian 09. Omitting these specifications in the integrations will provide energies that will not match Gaussian 16 results, even obtaining the LC $-\omega$ HPBE density functional. 


\section{References}

1. Li, G.; Zhang, D.; Qiao, Q.; Yu, Y.; Peterson, D.; Zafar, A.; Kumar, R.; Curtarolo, S.; Hunte, F.; Shannon, S.; Zhu, Y.; Yang, W.; Cao, L. All The Catalytic Active Sites of $\mathrm{MoS}_{2}$ for Hydrogen Evolution. Journal of the American Chemical Society 2016, 138, 16632-16638. doi:10.1021/jacs.6b05940.

2. Garrett, B.R.; Click, K.A.; Durr, C.B.; Hadad, C.M.; Wu, Y. $\left[\mathrm{MoO}\left(\mathrm{S}_{2}\right)_{2} \mathrm{~L}\right]^{1-}(\mathrm{L}=$ picolinate or pyrimidine-2-carboxylate) Complexes as $\mathrm{MoS}_{x}$-Inspired Electrocatalysts for Hydrogen Production in Aqueous Solution. Journal of the American Chemical Society 2016, 138, 13726-13731. doi:10.1021/jacs.6b08652.

3. Karunadasa, H.I.; Chang, C.J.; Long, J.R. A molecular molybdenum-oxo catalyst for generating hydrogen from water. Nature 2010, 464, 1329-1333. doi:10.1038/nature08969.

4. Bechlars, B.; D’Alessandro, D.M.; Jenkins, D.M.; Iavarone, A.T.; Glover, S.D.; Kubiak, C.P.; Long, J.R. High-spin ground states via electron delocalization in mixed-valence imidazolate-bridged divanadium complexes. Nature Chemistry 2010, 2, 362-368. doi:10.1038/nchem.585.

5. Yepes, D.; Jaque, P.; Martínez-Araya, J.I. Scrutinizing the substituent effect on Mo-based electrocatalysts for molecular hydrogen release through axial-equatorial decomposition: a DFT study. Phys. Chem. Chem. Phys. 2019, 21, 16601-16614. doi:10.1039/C9CP00670B.

6. Martínez-Araya, J.; Yepes, D.; Jaque, P. A 3D Visualization of the Substituent Effect. J. Mol. Model. 2018, 24, 31. doi:doi.org/10.1007/s00894-017-3565-8.

7. Sundstrom, E.J.; Yang, X.; Thoi, V.S.; Karunadasa, H.I.; Chang, C.J.; Long, J.R.; Head-Gordon, M. Computational and Experimental Study of the Mechanism of Hydrogen Generation from Water by a Molecular Molybdenum-Oxo Electrocatalyst. Journal of the American Chemical Society 2012, 134, 5233-5242. doi:10.1021/ja210949r.

8. Jun, L.; Kazunari, Y. Computational Evidence for Hydrogen Generation by Reductive Cleavage of Water and $\alpha-\mathrm{H}$ Abstraction on a Molybdenum Complex. Angewandte Chemie International Edition 2011, 50, 11972-11975, [https://onlinelibrary.wiley.com/doi/pdf/10.1002/anie.201102917]. doi:10.1002/anie.201102917.

9. Chermette, H. Chemical Reactivity Indexes in Density Functional Theory. Journal of Computational Chemistry 1999, 20, 129-154. doi:10.1002/(SICI)1096-987X(19990115)20:1<129::AID-JCC13>3.0.CO;2-A.

10. Geerlings, P.; De Proft, F.; Langenaeker, W. Conceptual Density Functional Theory. Chemical Reviews 2003, 103, 1793-1874. doi:10.1021/cr990029p.

11. Frau, J.; Muñoz, F.; Glossman-Mitnik, D. A Molecular Electron Density Theory Study of the Chemical Reactivity of Cis- and Trans-Resveratrol. Molecules 2016, 21, 1650. doi:10.3390/molecules21121650.

12. Frau, J.; Glossman-Mitnik, D. A conceptual DFT study of the molecular properties of glycating carbonyl compounds. Chemistry Central Journal 2017, 11, 8. doi:10.1186/s13065-017-0239-7.

13. Frau, J.; Glossman-Mitnik, D. Conceptual DFT Descriptors of Amino Acids with Potential Corrosion Inhibition Properties Calculated with the Latest Minnesota Density Functionals. Frontiers in Chemistry 2017, 5, 16. doi:10.3389/fchem.2017.00016.

14. Frau, J.; Glossman-Mitnik, D. Conceptual DFT Study of the Local Chemical Reactivity of the Colored BISARG Melanoidin and Its Protonated Derivative. Frontiers in Chemistry 2018, 6, 136. doi:10.3389/fchem.2018.00136.

15. Sousa, S.F.; Fernandes, P.A.; Ramos, M.J. General Performance of Density Functionals. The Journal of Physical Chemistry A 2007, 111, 10439-10452, [https://doi.org/10.1021/jp0734474]. PMID: 17718548, doi:10.1021/jp0734474.

16. Peverati, R.; Truhlar, D.G. Quest for a Universal Density Functional: The Accuracy of Density Functionals Across a Broad Spectrum of Databases in Chemistry and Physics. Philosophical Transactions of the Royal Society of London A: Mathematical, Physical and Engineering Sciences 2014, 372, 20120476. doi:10.1098/rsta.2012.0476.

17. Mardirossian, N.; Head-Gordon, M. Thirty years of density functional theory in computational chemistry: an overview and extensive assessment of 200 density functionals. Molecular Physics 2017, 115, 2315-2372, [https:/ / doi.org/10.1080/00268976.2017.1333644]. doi:10.1080/00268976.2017.1333644.

18. Pueyo Bellafont, N.; Illas, F.; Bagus, P.S. Validation of Koopmans' theorem for density functional theory binding energies. Phys. Chem. Chem. Phys. 2015, 17, 4015-4019. doi:10.1039/C4CP05434B. 
19. Frau, J.; Flores-Holguín, N.; Glossman-Mitnik, D. Chemical Reactivity Properties, pKa Values, AGEs Inhibitor Abilities and Bioactivity Scores of the Mirabamides A-H Peptides of Marine Origin Studied by Means of Conceptual DFT. Marine Drugs 2018, 16. doi:10.3390/md16090302.

20. Koopmans, T. Über die Zuordnung von Wellenfunktionen und Eigenwerten zu den Einzelnen Elektronen Eines Atoms. Physica 1933, 18, 104-113. doi:10.1016/S0031-8914(34)90011-2.

21. Zevallos, J.; Toro-Labbé, A. A Theoretical Analysis of the Kohn-Sham and Hartree-Fock Orbitals and the use in the Determination of Electronic Properties. Journal of the Chilean Chemical Society 2003, 48, $39-47$. doi:10.4067/S0717-97072003000400007.

22. Kronik, L.; Stein, T.; Refaely-Abramson, S.; Baer, R. Excitation Gaps of Finite-Sized Systems from Optimally Tuned Range-Separated Hybrid Functionals. Journal of Chemical Theory and Computation 2012, 8, 1515-1531, [https://doi.org/10.1021/ct2009363]. PMID: 26593646, doi:10.1021/ct2009363.

23. Janak, J.F. Proof that $\frac{\partial E}{\partial n_{i}}=\epsilon$ in Density-Functional Theory. Physical Review B 1978, 18, 7165-7168. doi:10.1103/PhysRevB.18.7165.

24. Tecmer, P.; Gomes, A.S.P.; Ekström, U.; Visscher, L. Electronic spectroscopy of UO22+, NUO+ and NUN: an evaluation of time-dependent density functional theory for actinides. Phys. Chem. Chem. Phys. 2011, 13, 6249-6259. doi:10.1039/C0CP02534H.

25. Frisch, M.J.; Trucks, G.W.; Schlegel, H.B.; Scuseria, G.E.; Robb, M.A.; Cheeseman, J.R.; Scalmani, G.; Barone, V.; Mennucci, B.; Petersson, G.A.; Nakatsuji, H.; Caricato, M.; Li, X.; Hratchian, H.P.; Izmaylov, A.F.; Bloino, J.; Zheng, G.; Sonnenberg, J.L.; Hada, M.; Ehara, M.; Toyota, K.; Fukuda, R.; Hasegawa, J.; Ishida, M.; Nakajima, T.; Honda, Y.; Kitao, O.; Nakai, H.; Vreven, T.; Montgomery, Jr., J.A.; Peralta, J.E.; Ogliaro, F.; Bearpark, M.; Heyd, J.J.; Brothers, E.; Kudin, K.N.; Staroverov, V.N.; Kobayashi, R.; Normand, J.; Raghavachari, K.; Rendell, A.; Burant, J.C.; Iyengar, S.S.; Tomasi, J.; Cossi, M.; Rega, N.; Millam, J.M.; Klene, M.; Knox, J.E.; Cross, J.B.; Bakken, V.; Adamo, C.; Jaramillo, J.; Gomperts, R.; Stratmann, R.E.; Yazyev, O.; Austin, A.J.; Cammi, R.; Pomelli, C.; Ochterski, J.W.; Martin, R.L.; Morokuma, K.; Zakrzewski, V.G.; Voth, G.A.; Salvador, P.; Dannenberg, J.J.; Dapprich, S.; Daniels, A.D.; Farkas, O.; Foresman, J.B.; Ortiz, J.V.; Cioslowski, J.; Fox, D.J. Gaussian 09 Revision E.01. Gaussian Inc. Wallingford CT 2009.

26. Frisch, M.J.; Trucks, G.W.; Schlegel, H.B.; Scuseria, G.E.; Robb, M.A.; Cheeseman, J.R.; Scalmani, G.; Barone, V.; Petersson, G.A.; Nakatsuji, H.; Li, X.; Caricato, M.; Marenich, A.V.; Bloino, J.; Janesko, B.G.; Gomperts, R.; Mennucci, B.; Hratchian, H.P.; Ortiz, J.V.; Izmaylov, A.F.; Sonnenberg, J.L.; Williams-Young, D.; Ding, F.; Lipparini, F.; Egidi, F.; Goings, J.; Peng, B.; Petrone, A.; Henderson, T.; Ranasinghe, D.; Zakrzewski, V.G.; Gao, J.; Rega, N.; Zheng, G.; Liang, W.; Hada, M.; Ehara, M.; Toyota, K.; Fukuda, R.; Hasegawa, J.; Ishida, M.; Nakajima, T.; Honda, Y.; Kitao, O.; Nakai, H.; Vreven, T.; Throssell, K.; Montgomery, Jr., J.A.; Peralta, J.E.; Ogliaro, F.; Bearpark, M.J.; Heyd, J.J.; Brothers, E.N.; Kudin, K.N.; Staroverov, V.N.; Keith, T.A.; Kobayashi, R.; Normand, J.; Raghavachari, K.; Rendell, A.P.; Burant, J.C.; Iyengar, S.S.; Tomasi, J.; Cossi, M.; Millam, J.M.; Klene, M.; Adamo, C.; Cammi, R.; Ochterski, J.W.; Martin, R.L.; Morokuma, K.; Farkas, O.; Foresman, J.B.; Fox, D.J. Gaussian 16 Revision B.01, 2016. Gaussian Inc. Wallingford CT.

27. Austin, A.; Petersson, G.A.; Frisch, M.J.; Dobek, F.J.; Scalmani, G.; Throssell, K. A Density Functional with Spherical Atom Dispersion Terms. Journal of Chemical Theory and Computation 2012, 8, 4989-5007, [https://doi.org/10.1021/ct300778e]. PMID: 26593191, doi:10.1021/ct300778e.

28. Fuentealba, P.; Preuss, H.; Stoll, H.; Szentpály, L.V. A proper account of core-polarization with pseudopotentials: single valence-electron alkali compounds. Chemical Physics Letters 1982, 89, 418-422. doi:http:/ /dx.doi.org/10.1016/0009-2614(82)80012-2.

29. von Szentpály, L.; Fuentealba, P.; Preuss, H.; Stoll, H. Pseudopotential calculations on $\mathrm{Rb}_{2}^{+}$, $\mathrm{Cs}_{2}^{+}, \mathrm{RbH}^{+}, \mathrm{CsH}^{+}$and the mixed alkali dimer ions. Chemical Physics Letters 1982, 93, 555-559. doi:http:/ /dx.doi.org/10.1016/0009-2614(82)83728-7.

30. Fuentealba, P.; Stoll, H.; von Szentpály, L.; Schwerdtfeger, P.; Preuss, H. On the reliability of semi-empirical pseudopotentials: simulation of Hartree-Fock and Dirac-Fock results. Journal of Physics B: Atomic and Molecular Physics 1983, 16, L323.

31. Stoll, H.; Fuentealba, P.; Schwerdtfeger, P.; Flad, J.; v. Szentpály, L.; Preuss, H. Cu and Ag as one-valence-electron atoms: $\mathrm{CI}$ results and quadrupole corrections for $\mathrm{Cu}_{2}, \mathrm{Ag}_{2}, \mathrm{CuH}$, and $\mathrm{AgH}$. The Journal of Chemical Physics 1984, 81, 2732-2736, [http:/ / dx.doi.org/10.1063/1.447992]. doi:10.1063/1.447992. 
32. Ditchfield, R.; Hehre, W.J.; Pople, J.A. Self-Consistent Molecular-Orbital Methods. IX. An Extended Gaussian-Type Basis for Molecular-Orbital Studies of Organic Molecules. The Journal of Chemical Physics 1971, 54, 724-728, [http:/ /dx.doi.org/10.1063/1.1674902]. doi:10.1063/1.1674902.

33. Hehre, W.J.; Ditchfield, R.; Pople, J.A. Self-Consistent Molecular Orbital Methods. XII. Further Extensions of Gaussian-Type Basis Sets for Use in Molecular Orbital Studies of Organic Molecules. The Journal of Chemical Physics 1972, 56, 2257-2261, [http:/ / dx.doi.org/10.1063/1.1677527]. doi:10.1063/1.1677527.

34. Rassolov, V.A.; Pople, J.A.; Ratner, M.A.; Windus, T.L. 6-31G* basis set for atoms K through Zn. The Journal of Chemical Physics 1998, 109, 1223-1229, [http:/ / dx.doi.org/10.1063/1.476673]. doi:10.1063/1.476673.

35. Clark, T.; Chandrasekhar, J.; Spitznagel, G.W.; Schleyer, P.V.R. Efficient diffuse function-augmented basis sets for anion calculations. III. The 3-21+G basis set for first-row elements, Li-F. Journal of Computational Chemistry 1983, 4, 294-301. doi:10.1002/jcc.540040303.

36. Wachters, A. Gaussian Basis Set for Molecular Wavefunctions Containing Third-Row Atoms. Physics 1970, 52, 1033-1036. doi:10.1063/1.1673095.

37. Krishnan, R.; Binkley, J.; Seeger, R.; Pople, J. Self-consistent molecular orbital methods. XX. A basis set for correlated wave functions. The Journal of Chemical Physics 1980, 72, 650-654. doi:10.1063/1.438955.

38. Morell, C.; Grand, A.; Toro-Labbé, A. New Dual Descriptor for Chemical Reactivity. The Journal of Physical Chemistry A 2005, 109, 205-212. doi:10.1021/jp046577a.

39. Morell, C.; Grand, A.; Toro-Labbé, A. Theoretical Support for using the $\Delta f(\mathbf{r})$ Descriptor. Chemical Physics Letters 2006, 425, 342-346. doi:10.1016/j.cplett.2006.05.003.

40. Martínez-Araya, J.I. Why is the Dual Descriptor a More Accurate Local Reactivity Descriptor than Fukui Functions? Journal of Mathematical Chemistry 2015, 53, 451-465. doi:10.1007/s10910-014-0437-7. 